\title{
Usefulness of the forced oscillation technique in assessing the therapeutic result of
}

\section{tracheobronchial central airway obstruction}

Short title: Usefulness of FOT in central airway obstruction

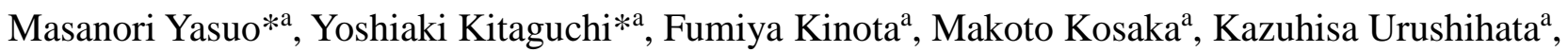
Atsuhito Ushiki ${ }^{\mathrm{a}}$, Hiroshi Yamamoto ${ }^{\mathrm{a}}$, Satoshi Kawakami ${ }^{\mathrm{b}}$, and Masayuki Hanaoka ${ }^{\mathrm{a}}$

${ }^{a}$ The First Department of Internal Medicine, Shinshu University School of Medicine, 3-1-1 Asahi

Matsumoto, 390-8621, Japan

b Department of Radiology, Shinshu University School of Medicine, 3-1-1 Asahi Matsumoto, 3908621, Japan

* Both of these authors contributed equally to this work.

E-mail address of each author:

Masanori Yasuo: yasumasa@shinshu-u.ac.jp

Yoshiaki Kitaguchi: kitaguti@shinshu-u.ac.jp

Fumiya Kinota: kinota@shinshu-u.ac.jp

Makoto Kosaka: kosaka@shinshu-u.ac.jp

Kazuhisa Urushihata: ichiju@shinshu-u.ac.jp 
Atsuhito Ushiki: atsuhito@shinshu-u.ac.jp

Hiroshi Yamamoto: yama5252@shinshu-u.ac.jp

Satoshi Kawakami: kawasato@shinshu-u.ac.jp

Masayuki Hanaoka: masayuki@shinshu-u.ac.jp

\section{Corresponding author:}

Masanori Yasuo, MD, PhD

The First Department of Internal Medicine

Shinshu University School of Medicine

3-1-1 Asahi, Matsumoto, 390-8621, JAPAN

Tel: +81 26337 2631, Fax: +81 263363722

E-mail address: yasumasa@shinshu-u.ac.jp 


\section{Abstract}

Background: Pulmonary function tests (PFTs) comprise the traditional method for detecting central airway obstruction (CAO) and evaluating therapeutic effects, but are effort-dependent. By contrast, the forced oscillation technique (FOT) is performed during tidal breathing in an effort-independent mode and is universally used to assess respiratory function in patients with chronic obstructive pulmonary disease (COPD) and asthma. We used the FOT to measure airway resistance and reactance in patients with CAO before and after interventional bronchoscopy and compared the results to data obtained using PFTs.

Methods: Twelve patients with CAO were recruited from December 2013 to July 2016. The FOT, PFTs, chest computed tomography (CT), COPD Assessment Test (CAT), and the modified Medical Research Council (mMRC) dyspnea scale were employed before and after interventional bronchoscopy. The minimum airway cross-sectional area (MACSA) was calculated using a CT image calculator.

Results: Of the 12 patients, 6 had tracheal obstruction and 6 had bronchial obstruction. All FOT measurements, except $\Delta \mathrm{X} 5$, were significantly improved after interventional bronchoscopy in all cases. The significance of the improvement was greater with the FOT than PFTs. The MACSA, CAT, and mMRC dyspnea scale scores also significantly improved in all cases. Furthermore, only alteration of resistance at $20 \mathrm{~Hz}$ (R20) significantly correlated with the alteration of the MACSA after intervention. No significant correlations were found for PFTs. 
Conclusions: The FOT is suitable and convenient for assessing therapeutic results in patients with tracheobronchial CAO. The alteration of R20 is useful for estimating the airway dilation of CAO after interventional bronchoscopy.

Keywords: Forced oscillation technique (FOT), Interventional bronchoscopy, Pulmonary function test (PFT), Therapeutic effect 


\section{List of abbreviations}

$\mathrm{CAO}=$ central airway obstruction

CAT $=$ COPD Assessment Test

$\mathrm{CT}=$ computed tomography

FOT $=$ forced oscillation technique

mMRC $=$ modified Medical Research Council

MACSA $=$ minimum airway cross-sectional area

PFT = pulmonary function test 


\section{Introduction}

Pulmonary function tests (PFTs) are widely used to detect central airway obstruction (CAO)

and assess treatment results. However, PFTs are unsuitable for patients with severe tracheobronchial CAO because of its effort dependence. Tracheobronchial CAO can have benign or malignant causes.

The therapy for tracheobronchial CAO is also varied, including surgery, interventional bronchoscopy, and radiotherapy.

Recently, a broadband frequency forced oscillation technique (FOT) has been applied frequently for the measurement of lung function and the evaluation of pulmonary disease status, particularly chronic obstructive pulmonary disease (COPD) and asthma, because of its effort independence in demanding minimal cooperation from patients [1]. The FOT was originally reported in the 1950s [2]. In this technique, random pressure pulses of 5-35 Hz are generated by a small loudspeaker mounted in series with a pneumotachograph. These pulses are applied to a patient during tidal breathing. Pressure-flow oscillations are superimposed on the patient's tidal breaths and real-time recordings are used to estimate the total respiratory system impedance, including resistance 
and reactance, at different frequencies that may differentiate between central and peripheral airway obstruction [3].

Several researchers have tried to apply the FOT to assess CAO [4-6]. All of this research has used impulse oscillometry (IOS) (MasterScreen IOS, Jaeger, Hoechberg, Germany) to assess the therapeutic effect of the FOT and estimate the usefulness of follow-up [4], degree of tracheal stenosis [5], and correlation of the symptom improvements with the type of stenosis [6] in patients with tracheal or upper airway obstruction.

In the current study, we applied the FOT to the measurement of airway resistance in patients with CAO before and after interventional bronchoscopy to evaluate the therapeutic effects, which were then verified using the corresponding data obtained using PFTs. In addition, we evaluated the correlation of FOT measurements with the airway dilation of CAO after interventional bronchoscopy.

\section{Patients and Methods}

This study was approved by the Ethics Review Board of Shinshu University (Permission number: 3505, Date of approval: September 6, 2016). The study protocols and interventional bronchoscopy were performed in accordance with the principles outlined in the Declaration of Helsinki of the World Medical Association. Before the study and interventional bronchoscopy, written informed consent was obtained from each patient.

\subsection{Patients}


Our institute is a major medical facility for the treatment of patients with CAO with interventional bronchoscopy in Nagano prefecture in Japan. Tracheobronchial CAO was defined as CAO with various causes (e.g., benign, malignant, iatrogenic, inflammatory). Tracheobronchial CAO was diagnosed using chest computed tomography (CT) and bronchoscopy. From December 2013 to July 2016, 23 patients with CAO were treated using interventional bronchoscopy, including 12 patients (9 males, 3 females) with CAO who systematically underwent the FOT, respiratory spirometry, modified Medical Research Council (mMRC) questionnaire for dyspnea assessment, COPD Assessment Test (CAT), and chest CT before and after interventional bronchoscopy. Of these 12 patients, 8 had malignant obstruction and 4 had benign causes of CAO (Table 1). According to the position of the obstruction, the 12 patients were further divided into those with tracheal obstruction $(n=6)$ and main bronchial obstruction $(n=6)$. Regarding the therapeutic procedure, 5 patients underwent endobronchial intervention (e.g., electrosurgical snare, argon plasma coagulation) and the other 7 patients underwent endobronchial intervention with stent insertion (1 silicone and 6 metallic stents; Table 1). All of the patients exhibited respiratory symptoms, such as coughing, dyspnea, and stridor. In this study, the central airway was defined as the trachea to the left main bronchus and bronchus intermedius in the right-side bronchus.

\subsection{Measurements}


The FOT, PFTs, chest CT, mMRC dyspnea scale, and CAT were performed for the 12 patients with CAO within 5 days before and 7 days after interventional bronchoscopy to avoid the stages of luminal edema and excessive secretion of blood and mucus.

\subsubsection{FOT}

Before any other PFT, the FOT was applied to measure the respiratory impedance using a commercially available multi-frequency FOT device (MostGraph-01, Chest Co., Ltd., Tokyo, Japan) following the standard recommendations [12] as described previously [1, 11]. The resistance at $5 \mathrm{~Hz}$ (R5), resistance at $20 \mathrm{~Hz}$ (R20), reactance at $5 \mathrm{~Hz}$ (X5), resonant frequency (Fres), and low-frequency reactance area (ALX) were measured. The oscillatory parameters were measured at whole-breath, inspiratory, and expiratory phases. The difference between the inspiratory and expiratory phases was calculated for each oscillatory parameter.

\subsubsection{PFT}

The PFTs were performed by 2 PFT technicians according to the American Thoracic Society criteria. As described previously [7], spirometry, the diffusion capacity of the lung for carbon monoxide (DLco), closing volume, and a global measurement of ventilation heterogeneity (the slope of phase III of the single breath nitrogen washout test [delta $\mathrm{N}_{2}$ ]) were measured using a pulmonary function testing system (Chestac-8800, Chest Co., Ltd.). The predicted values for forced expiratory volume in $1 \mathrm{~s}\left(\mathrm{FEV}_{1}\right)$ and vital capacity were derived according to standard data published by the Japanese Respiratory Society [8]. The predicted values for DLco, DLco/alveolar volume, and lung 
volumes (RV and TLC) were determined using the formulas described by Nishida et al. [9] and Boren et al. [10]. Tests were repeated 2 or 3 times to confirm repeatability.

\subsection{3 mMRC scale}

The mMRC scale is the most commonly used validated scale for chronic respiratory diseases to assess dyspnea in daily living [13]. Dyspnea was evaluated in patients using the mMRC scale, which consists of 5 statements that describe almost the entire range of dyspnea from none (Grade 0 ) to almost complete incapacity (Grade 4).

\subsubsection{CAT}

The CAT is one of the questionnaires used to evaluate the health-related quality of life in patients with COPD [14] and other chronic lung diseases $[15,16]$. The questionnaire consists of 8 items: cough, phlegm, chest tightness, breathlessness, going up hills/stairs, limitation of activity at home, confidence in leaving the house, and sleep and energy. The score ranges from 0 (best) to 40 (worst) points. All of the patients were evaluated for health-related quality of life using the CAT.

\subsubsection{Minimum airway cross-sectional area}

The minimum airway cross-sectional area (MACSA) was measured using CT image customizable software (DICOM viewer-EV Insite R; PSP corporation, Tokyo, Japan). The most obstructed area was manually indicated on the image. If the most obstructed area was indicated obliquely, the image was reconstructed to show the most obstructed area horizontally. The MACSA was then calculated by tracing the most obstructed area in EV Insite R (Figure 1). 


\subsection{Bronchoscopy}

Interventional bronchoscopy was performed using a bronchoscope (6.0-mm outer diameter of the tip; TYPE 1T260, Olympus, Tokyo, Japan) in a room equipped with a radiographic fluoroscopy apparatus. The routine bronchoscopy maneuvers and interventional bronchoscopy procedures used at our institution have been described elsewhere $[17,18]$.

\subsection{Statistical analysis}

Statistical analyses were performed using the Statistical Package for the Social Sciences (SPSS) for Windows software program (version 14.0, SPSS Inc., Chicago, IL, USA). The mMRC scale and CAT scores were presented as the median and quartile. The other quantitative values were presented as the mean \pm standard error of the mean. The Wilcoxon signed-rank test was used to compare the mMRC scale and CAT results before and after interventional bronchoscopy in patients with CAO. The paired t-test was used to compare the other results. In addition, the correlation between the FOT measurements and the dilation of the CAO treated by interventional bronchoscopy was evaluated using Pearson’s product correlation. P-values $<0.05$ were considered significant.

\section{Results}

\subsection{Interventional bronchoscopy}

As shown in Table 2, the MACSA was significantly improved by interventional bronchoscopy in the 12 patients with CAO $\left(35.33 \pm 6.97 \mathrm{~mm}^{2}\right.$ vs. $\left.120.87 \pm 20.33 \mathrm{~mm}^{2}, \mathrm{P}<0.01\right)$, as well as in the tracheal 
obstruction (38.39 $\pm 6.28 \mathrm{~mm}^{2}$ vs. $\left.121.85 \pm 28.30 \mathrm{~mm}^{2}, \mathrm{P}<0.01\right)$ and bronchial obstruction $(32.28 \pm$ $13.06 \mathrm{~mm}^{2}$ vs. $\left.119.90 \pm 31.90 \mathrm{~mm}^{2}, \mathrm{P}<0.05\right)$ subgroups. The mMRC dyspnea scale and CAT scores for the 12 patients with CAO were also significantly improved by interventional bronchoscopy (mMRC: $2.41 \pm 0.31$ vs. $1.33 \pm 0.31, \mathrm{P}<0.01$; CAT: $18.50 \pm 3.46$ vs. $13.13 \pm 2.74, \mathrm{P}<0.05$ ); however, these scores did not significantly improve after interventional bronchoscopy in the subgroup analysis, probably because of the small number of patients.

\subsection{FOT}

As shown in Table 3, the FOT measurements in the 12 patients with CAO (R5, R20, R5-R20, X5,

Fres, and ALX) were significantly improved by interventional bronchoscopy in terms of the whole breath, expiratory phase, and inspiratory phase $(\mathrm{P}<0.01)$. The measurements were also significantly improved by interventional bronchoscopy in the tracheal obstruction subgroup $(\mathrm{P}<0.01)$ in the expiratory phase, but only R5 and R20 were significantly improved by interventional bronchoscopy in the bronchial obstruction subgroup in terms of the whole breath, expiratory phase, and inspiratory phase.

\subsection{PFT}

Most of the critical parameters of the PFTs were significantly improved by interventional bronchoscopy in the 12 patients with CAO (Table 4). Only a few PFT parameters were significantly 
improved by interventional bronchoscopy in the tracheal obstruction and bronchial obstruction subgroups (Table 4). FRC, RV, and DLco were not significantly improved (Supplemental Table 1).

\subsection{Correlation of altered MACSA with alterations in the FOT, PFTs, mMRC, and CAT results}

\section{before and after interventional bronchoscopy}

Alteration of the MACSA was significantly correlated with alterations in R20 before and after interventional bronchoscopy in terms of the whole breath, expiratory phase, and inspiratory phase in patients with $\mathrm{CAO}(\mathrm{r}=0.61$ for whole breath, $\mathrm{r}=0.61$ for expiratory phase, and $\mathrm{r}=0.60$ for inspiratory phase, all $\mathrm{P}<0.05$, Figure 2, Supplemental Table 2). The alteration of the MACSA before and after interventional bronchoscopy was not significantly correlated with alterations in mMRC, CAT, and PFT results before and after interventional bronchoscopy in patients with CAO (Supplemental Table 2).

\section{Discussion}

The results demonstrated that the FOT may be more reliable than ordinal PFTs for estimating the effects of interventional bronchoscopy on the management of CAO. The most attractive advantage of the FOT in CAO patients is its effort independence $[4,6]$. Not all of the CAO patients could tolerate PFTs because of their effort dependency. Because this study was designed to compare the results of the FOT and PFTs for CAO, we had to select patients who could tolerate both. Among the 23 patients with CAO, 11 could not perform the PFTs because of the severity of their disease. 
Assessing pulmonary function in CAO patients is difficult because the disease status is sometimes so severe that the patients cannot endure the PFTs for evaluation of the degree of airway obstruction before treatment. Three previous investigations assessed the usefulness of the FOT in CAO patients [4-6], including post-tracheostomy tracheal stenosis caused by central nervous system injuries [4] and 20 patients with tracheal obstruction [6], using MasterScreen IOS® (Jaeger). In the present study, the CAO patients included both those with tracheal obstruction and those with bronchial obstruction, and the assessment apparatus was the MostGraph-01 (Chest Co., Ltd.). This is the first report using the MostGraph-01 to assess airway resistance in CAO patients.

The other effort-independent method for estimating airway obstruction is CT. With the recent improvement of high resolution CT, precise information on airway obstruction can be obtained, with the advantage of calculation of the degree of airway obstruction (such as the MACSA in this study). The FOT had some advantages over CT for the CAO patients; first, some of the CAO patients could not maintain the supine position in the gantry of the CT scanner because of airway suffocation. Second, although we used the MACSA for estimating the improvement of airway dilation, no standardized index exists for estimating the degree of airway obstruction in CT. Third, frequently undergoing CT amplifies exposure to radiation. Fourth, despite being widely used in developed countries (e.g., Japan), CT scanners are not easily accessible in some medical institutes in undeveloped and developing countries. Lastly, the FOT apparatus is much cheaper than CT scanners in clinical practice.

Compared to the PFT results of the entire cohort $(n=12$; Table 4$)$, the FOT produced larger and 
more significant improvements (Table 3). No significant improvements were observed in the minute pulmonary functions except TLC (Supplemental Table 1). Analysis of the correlation of the MACSA with the FOT, PFT, mMRC scale, and CAT results revealed that only R20 from the FOT was significantly correlated with the MACSA (Supplemental Table 2). On the basis of these results, the FOT seems to be more sensitive in detecting an effect of airway dilation in CAO treated using interventional bronchoscopy.

The results of the FOT for CAO were similar to those reported in patients with COPD [5, 6, 19]. Previous studies $[1,7,20]$ have shown increased resistance at $5 \mathrm{~Hz}$, marked frequency dependence of resistance, more negative reactance at $5 \mathrm{~Hz}$, and increased Fres. Comparing the FOT results for CAO and COPD [1, 7, 20], we also found relatively high resistance at $5 \mathrm{~Hz}$ and $20 \mathrm{~Hz}$, increased Fres and ALX, and more negative reactance at $5 \mathrm{~Hz}$.

Compared to tracheal obstruction before interventional bronchoscopy, the airway resistance was relatively low in main bronchial obstruction. R5 and R20 were significantly improved in main bronchial obstruction after interventional bronchoscopy, regardless of the breathing phase (Table 3). In tracheal obstruction after interventional bronchoscopy, significant improvement of all FOT components was observed in the expiratory phase (Table 3). The expiratory phase of the FOT is the most sensitive for estimating improvements after interventional bronchoscopy. In contrast, some components of conventional PFTs improved, particularly in tracheal obstruction after interventional bronchoscopy; however, the number of improved components and degree of significance were lower 
for conventional PFTs than for the FOT (Table 4). Compared with the tracheal obstruction group, the 6 cases of bronchial obstruction did not show significant changes in conventional lung function test results after intervention except the parameter of air flow rate at 50\% vital capacity, which might indicate the limitation of conventional PFTs performed on CAO patients with bronchial obstruction (i.e., effort dependency). The P-values for slow vital capacity, forced vital capacity, FEV1, peak expiratory flow rate (PEFR), and the air flow rate at 25\% vital capacity in the bronchial obstruction group were all over 0.1. However, MMF and PEFR, had P-values of 0.142 in the bronchial obstruction group, which implies that these parameters might show significant improvement if the sample size were larger. By contrast, R5 and R20 presented significant changes in the bronchial obstruction group after interventional bronchoscopy. Additionally, the MACSA and mMRC dyspnea scale scores also significantly improved in the bronchial obstruction group after interventional bronchoscopy (Table 2). Together, these results suggest that the FOT is more sensitive than conventional PFTs for estimating the therapeutic effect on CAO.

This study has some limitations. First, the sample size was small. CAO is rare, with even fewer patients capable of completing the FOT, PFT, mMRC questionnaire, and CAT before and after interventional bronchoscopy. Second, we used the CAT to estimate health-related quality of life in patients with CAO, but the CAT has not yet been validated for CAO. It would be impossible to determine validity and reproducibility in the same CAO patient because of the fluctuation in symptoms. 


\section{Conclusions}

The FOT is an effort-independent test that can be performed in patients with CAO to estimate the treatment effect. The R20 parameter of FOT is significantly improved by the effects of interventional bronchoscopy for airway dilation. Therefore, the FOT should be applied to patients with CAO to assess the therapeutic effect on tracheobronchial CAO. 


\section{Financial/nonfinancial disclosures}

There is no funding source to declare.

\section{Conflict of interest}

The authors have no conflicts of interest to disclose.

\section{Acknowledgements}

The authors would like to thank Dr. Yunden Droma for her valuable advice and for kindly checking the English usage in our manuscript. 


\section{References}

[1] Shirai T, Kurosawa H. Clinical application of the forced oscillation technique. Intern Med 2016;55:559-66.

[2] Dubois AB, Brody AW, Lewis DH, Burgess BF Jr. Oscillation mechanics of lung and chest in man. J Appl Physiol 1956;8:587-94.

[3] Smith HJ, Reinhold P, Goldman MD. Forced oscillation technique and impulse oscillometry. Eur Respir Mon 2005;31:72-105.

[4] Horan T, Mateus S, Beraldo P, Araújo L, Urschel J, Urmenyi E, Santiago F. Forced oscillation technique to evaluate tracheostenosis in patients with neurologic injury. Chest 2001;120:69-73.

[5] Verbanck S, de Keukeleire T, Schuermans D, Meysman M, Vincken W, Thompson B. Detecting upper airway obstruction in patients with tracheal stenosis. J Appl Physiol 2010;109:47-52.

[6] Handa H, Huang J, Murgu SD, Mineshita M, Kurimoto N, Colt HG, et al. Assessment of 
central airway obstruction using impulse oscillometry before and after interventional bronchoscopy. Respir Care 2014;59:231-40.

[7] Kitaguchi Y, Yasuo M, Hanaoka M. Comparison of pulmonary function in patients with COPD, asthma-COPD overlap syndrome, and asthma with airflow limitation. Int J Chron Obstruct Pulmon Dis 2016;11:991-7.

[8] Japanese Society of Chest Disease. Standards of pulmonary function tests for Japanese. Jpn J Respir Dis 1993;31:421-7.

[9] Nishida O, Kambe M, Sewake N, Takano M, Kawane H. Pulmonary function in healthy subjects and its prediction: 5. Pulmonary diffusing capacity in adults. Jpn J Clin Pathol 1976;24:941-7.

[10]Boren HG, Kory RC, Syner JC, Callahan R. The Veterans Administration-Army cooperative study of pulmonary function. 2. The lung volume and its subdivisions in normal men. Am J Med 1966;41:96-101.

[11]Mori K, Shirai T, Mikamo M, Shishido Y, Akita T, Morita S, et al. Colored 3-dimensional analyses of respiratory resistance and reactance in COPD and asthma. COPD 2011;8:45663.

[12] Oostveen E, MacLeod D, Lorino H, Farré R, Hantos Z, Desager K, et al. ERS Task Force on Respiratory Impedance Measurements. The forced oscillation technique in clinical practice: methodology, recommendations and future developments. Eur Respir J 2003;22:1026-41. 
[13]Perez T, Burgel PR, Paillasseur JL, Caillaud D, Deslée G, Chanez P, et al; INITIATIVES BPCO Scientific Committee. Modified medical research council scale vs baseline dyspnea index to evaluate dyspnea in chronic obstructive pulmonary disease. Int J Chron Obstruct Pulmon Dis 2015;10:1663-72.

[14]Jones PW, Harding G, Berry P, Wiklund I, Chen WH, Kline Leidy N. Development and first validation of the COPD Assessment Test. Eur Respir J 2009;34:648-54.

[15]Hama M, Ushiki A, Kosaka M, Yamazaki Y, Yasuo M, Yamamoto H, et al. Health-related quality of life in patients with pulmonary non-tuberculous mycobacteria infection. Int $\mathrm{J}$ Tuberc Lung Dis 2016;20:747-52.

[16]Nagata K, Tomii K, Otsuka K, Tachikawa R, Otsuka K, Takeshita J, et al. Evaluation of the chronic obstructive pulmonary disease assessment test for measurement of health-related quality of life in patients with interstitial lung disease. Respirology 2012;17:506-12.

[17] Yasuo M, Tanabe T, Tsushima K, Nakamura M, Kanda S, Komatsu Y, et al. Endobronchial argon plasma coagulation for the management of post-intubation tracheal stenosis. Respirology 2006;11:659-62.

[18]Shioi R, Yasuo M, Ushiki A, Tanabe T, Tsushima K, Hanaoka M, et al. Management of right upper airway patency by a silicon stent in a case of endobronchial metastasis. Respir Med CME 2009;2:191-6.

[19]Handa H, Miyazawa T, Murgu SD, Nishine H, Kurimoto N, Huang J, et al. Novel 
multimodality imaging and physiologic assessments clarify choke-point physiology and airway wall structure in expiratory central airway collapse. Respir Care 2012;57:634-41.

[20] Shiota S, Katoh M, Fujii M, Aoki S, Matsuoka R, Fukuchi Y. Predictive equations and the reliability of the impulse oscillatory system in Japanese adult subjects. Respirology 2005;10:310-5.

\section{Figure Legends}

Figure 1. Representative figure of the minimum airway cross-sectional area.

(A) Before intervention. (B) After intervention. The minimum airway cross-sectional area was 
calculated using computed tomography image customizable software. The obstructed area was traced and the area calculated automatically. The calculated area is indicated at the top of both images.

Yellow words (from above): enclosed area, average Hounsfield unit (HU), minimum HU, maximum $\mathrm{HU}$, and standard deviation in the area.

Figure 2. Relationship between the changes in the minimum airway cross-sectional area and changes in resistance at $20 \mathrm{~Hz}$

A significant relationship was observed only between the 2 factors of minimum airway crosssectional area and R20. Whole breath phase is indicated in the figure $(r=0.61, P<0.05)$. The other phases (i.e., expiratory and inspiratory) showed almost the same relationships. 
Table 1. Information on the patients, airway lesions, and therapeutic procedures

\begin{tabular}{|c|c|c|c|c|c|c|}
\hline $\begin{array}{l}\text { Case } \\
\text { No. }\end{array}$ & $\begin{array}{c}\text { Age } \\
\text { (years) }\end{array}$ & Sex & Cause of airway obstruction & $\begin{array}{l}\text { Smoking history } \\
\text { (pack/year) }\end{array}$ & Stenotic site & Therapy \\
\hline \multicolumn{7}{|c|}{ Central airway obstruction of the trachea } \\
\hline 1 & 65 & M & GPA & 22 & Upper trachea & Ballooning, APC \\
\hline 2 & 78 & M & Thyroid cancer & 60 & Upper trachea & EMS \\
\hline 3 & 62 & M & Inflammatory polyp & 33 & Middle trachea & Snare resection, APC \\
\hline 4 & 54 & $\mathrm{~F}$ & $\begin{array}{l}\text { Metastatic mediastinal lymph node from } \\
\text { leiomyosarcoma of the uterus }\end{array}$ & 0 & Lower trachea & EMS \\
\hline 5 & 66 & $\mathrm{~F}$ & Lung cancer (Ad.) & 0 & Lower trachea & EMS \\
\hline 6 & 64 & M & Posttracheostomy tracheal obstruction & 64.5 & Upper trachea & APC \\
\hline \multicolumn{7}{|c|}{ Central airway obstruction of the main bronchus } \\
\hline 7 & 70 & M & Esophageal cancer & 4.5 & Left main bronchus & EMS \\
\hline 8 & 73 & M & Renal cell carcinoma & 16 & $\begin{array}{l}\text { Carina to left main } \\
\text { bronchus }\end{array}$ & Y-shape Dumon stent \\
\hline 9 & 71 & M & Lung cancer (Sq.) & 48 & Right main bronchus & Snare resection, APC \\
\hline 10 & 60 & M & $\begin{array}{l}\text { Endobronchial metastasis of rectal } \\
\text { cancer }\end{array}$ & 7.5 & Left main bronchus & EMS \\
\hline 11 & 67 & $\mathrm{M}$ & Lung cancer (Sq.) & 46 & Right main bronchus & EMS \\
\hline 12 & 47 & $\mathrm{~F}$ & Bronchial Schwannoma & 85 & Right main bronchus & Snare resection, APC \\
\hline
\end{tabular}

M, male; F, female; GPA, granulomatosis with polyangiitis; Sq., squamous cell carcinoma; Ad., adenocarcinoma; APC, argon plasma coagulation; EMS, expandable metallic stent; No., number code. 
Table 2. Effects of interventional bronchoscopy on airway dilation and symptoms

\begin{tabular}{|c|c|c|c|c|c|c|}
\hline & \multicolumn{2}{|c|}{ Whole obstruction $(n=12)$} & \multicolumn{2}{|c|}{ Tracheal obstruction $(n=6)$} & \multicolumn{2}{|c|}{ Bronchial obstruction $(n=6)$} \\
\hline & Before & After & Before & After & Before & After \\
\hline $\operatorname{MACSA}\left(\mathrm{mm}^{2}\right)$ & $35.33 \pm 6.97$ & $120.87 \pm 20.33^{* *}$ & $38.39 \pm 6.28$ & $\begin{array}{l}121.85 \pm \\
28.30 * *\end{array}$ & $32.28 \pm 13.06$ & $119.90 \pm 31.90 *$ \\
\hline $\begin{array}{l}\text { mMRC dyspnea } \\
\text { scale }\end{array}$ & $2.5(1.75-3)$ & $1.0(0.75-2) * *$ & $2.50(1.25-3)$ & $1.0(1-1.75)$ & $2.50(2-3.75)$ & $\begin{array}{c}1.50(0.25-2.75) \\
*\end{array}$ \\
\hline CAT total score ${ }^{\S}$ & $16.0(11-28.25)$ & $10.0(8.25-16.75)$ & $11.5(11-14)$ & $9.5(8.25-10)$ & $\begin{array}{l}28.5(22.5- \\
29.5)\end{array}$ & $19.5(12-25)$ \\
\hline
\end{tabular}

$* * \mathrm{P}<0.01, * \mathrm{P}<0.05$ vs. before intervention.

MACSA, minimum airway cross-sectional area; mMRC, modified Medical Research Council; CAT, Chronic Pulmonary Obstructive Disease Assessment Test.

For the MACSA. values are presented as the mean \pm standard error of the mean.

For the mMRC and CAT total score, values are presented as the median. The interquartile range is indicated in parentheses. 
Table 3. Forced oscillation technique measurements (MostGraph-01) before and after treatment

\begin{tabular}{|c|c|c|c|c|c|c|}
\hline & \multicolumn{2}{|c|}{ Whole obstruction $(n=12)$} & \multicolumn{2}{|c|}{ Tracheal obstruction $(n=6)$} & \multicolumn{2}{|c|}{ Bronchial obstruction $(n=6)$} \\
\hline & Before & After & Before & After & Before & After \\
\hline \multicolumn{7}{|l|}{ (whole breath) } \\
\hline $\mathrm{R} 5\left(\mathrm{cmH}_{2} \mathrm{O} / \mathrm{L} / \mathrm{s}\right)$ & $6.36 \pm 0.64$ & $3.79 \pm 0.38 * *$ & $7.18 \pm 0.91$ & $3.63 \pm 0.53^{* *}$ & $5.54 \pm 0.83$ & $3.94 \pm 0.59 * *$ \\
\hline $\mathrm{R} 20\left(\mathrm{cmH}_{2} \mathrm{O} / \mathrm{L} / \mathrm{s}\right)$ & $4.67 \pm 0.29$ & $3.01 \pm 0.24^{* *}$ & $5.05 \pm 0.38$ & $3.00 \pm 0.33^{* *}$ & $4.30 \pm 0.42$ & $3.03 \pm 0.39 * *$ \\
\hline $\mathrm{R} 5-\mathrm{R} 20\left(\mathrm{cmH}_{2} \mathrm{O} / \mathrm{L} / \mathrm{s}\right)$ & $1.69 \pm 0.36$ & $0.78 \pm 0.17^{* *}$ & $2.14 \pm 0.55$ & $0.64 \pm 0.28 * *$ & $1.24 \pm 0.44$ & $0.91 \pm 0.21$ \\
\hline $\mathrm{X} 5\left(\mathrm{cmH}_{2} \mathrm{O} / \mathrm{L} / \mathrm{s}\right)$ & $-3.77 \pm 0.95$ & $-1.03 \pm 0.29 * *$ & $-5.44 \pm 1.50$ & $-0.72 \pm 0.33$ & $-2.09 \pm 0.80$ & $-1.34 \pm 0.46$ \\
\hline Fres $(\mathrm{Hz})$ & $19.73 \pm 2.84$ & $12.18 \pm 1.76^{* *}$ & $23.12 \pm 3.94$ & $10.61 \pm 2.26$ & $16.35 \pm 3.94$ & $13.75 \pm 2.75$ \\
\hline \multicolumn{7}{|l|}{ (Expiratory phase) } \\
\hline $\mathrm{R} 5\left(\mathrm{cmH}_{2} \mathrm{O} / \mathrm{L} / \mathrm{s}\right)$ & $6.81 \pm 0.69$ & $4.01 \pm 0.43^{* *}$ & $7.73 \pm 0.94$ & $3.79 \pm 0.60 * *$ & $5.88 \pm 0.92$ & $4.23 \pm 0.67^{* *}$ \\
\hline $\mathrm{R} 20\left(\mathrm{cmH}_{2} \mathrm{O} / \mathrm{L} / \mathrm{s}\right)$ & $4.88 \pm 0.31$ & $3.13 \pm 0.26^{* *}$ & $5.32 \pm 0.41$ & $3.10 \pm 0.37 * *$ & $4.44 \pm 0.42$ & $3.16 \pm 0.40^{* *}$ \\
\hline $\mathrm{R} 5-\mathrm{R} 20\left(\mathrm{cmH}_{2} \mathrm{O} / \mathrm{L} / \mathrm{s}\right)$ & $1.92 \pm 0.39$ & $0.88 \pm 0.21^{* *}$ & $2.41 \pm 0.54$ & $0.69 \pm 0.31^{* *}$ & $1.44 \pm 0.52$ & $1.08 \pm 0.28$ \\
\hline $\mathrm{X} 5\left(\mathrm{cmH}_{2} \mathrm{O} / \mathrm{L} / \mathrm{s}\right)$ & $-4.01 \pm 1.14$ & $-1.06 \pm 0.34^{*}$ & $-5.96 \pm 1.84$ & $-0.63 \pm 0.34^{* *}$ & $-2.07 \pm 0.90$ & $-1.49 \pm 0.55$ \\
\hline Fres $(\mathrm{Hz})$ & $19.90 \pm 3.07$ & $12.53 \pm 2.06^{* *}$ & $23.83 \pm 4.20$ & $10.49 \pm 2.56^{* *}$ & $15.96 \pm 4.19$ & $14.57 \pm 3.23$ \\
\hline $\mathrm{ALX}\left(\mathrm{cmH}_{2} \mathrm{O} / \mathrm{L} / \mathrm{s} \mathrm{Hz}\right)$ & $41.3 \pm 12.29$ & $8.30 \pm 2.93^{* *}$ & $62.27 \pm 20.07$ & $4.39 \pm 2.05^{* *}$ & $20.40 \pm 9.30$ & $12.22 \pm 5.23$ \\
\hline \multicolumn{7}{|l|}{ (Inspiratory phase) } \\
\hline $\mathrm{R} 5\left(\mathrm{cmH}_{2} \mathrm{O} / \mathrm{L} / \mathrm{s}\right)$ & $5.91 \pm 0.59$ & $3.56 \pm 0.34^{* *}$ & $6.64 \pm 0.89$ & $3.48 \pm 0.47 * *$ & $5.19 \pm 0.74$ & $3.64 \pm 0.53^{* *}$ \\
\hline $\mathrm{R} 20\left(\mathrm{cmH}_{2} \mathrm{O} / \mathrm{L} / \mathrm{s}\right)$ & $4.46 \pm 0.29$ & $2.89 \pm 0.24 * *$ & $4.77 \pm 0.37$ & $2.89 \pm 0.31^{* *}$ & $4.16 \pm 0.43$ & $2.90 \pm 0.40 * *$ \\
\hline $\mathrm{R} 5-\mathrm{R} 20\left(\mathrm{cmH}_{2} \mathrm{O} / \mathrm{L} / \mathrm{s}\right)$ & $1.45 \pm 0.35$ & $0.67 \pm 0.14^{* *}$ & $1.87 \pm 0.57$ & $0.60 \pm 0.24^{* *}$ & $1.03 \pm 0.35$ & $0.75 \pm 0.15$ \\
\hline $\mathrm{X} 5\left(\mathrm{cmH}_{2} \mathrm{O} / \mathrm{L} / \mathrm{s}\right)$ & $-3.52 \pm 0.81$ & $-1.00 \pm 0.24^{* *}$ & $-4.92 \pm 1.23$ & $-0.81 \pm 0.32$ & $-2.12 \pm 0.76$ & $-1.19 \pm 0.38$ \\
\hline Fres $(\mathrm{Hz})$ & $19.56 \pm 2.64$ & $11.82 \pm 1.49 * *$ & $22.40 \pm 3.70$ & $10.72 \pm 2.01$ & $16.73 \pm 3.71$ & $12.92 \pm 2.30$ \\
\hline $\mathrm{ALX}\left(\mathrm{cmH}_{2} \mathrm{O} / \mathrm{L} / \mathrm{s} \mathrm{Hz}\right)$ & $34.56 \pm 9.19$ & $6.21 \pm 1.80^{* *}$ & $50.75 \pm 14.59$ & $4.74 \pm 2.02$ & $18.38 \pm 7.38$ & $7.68 \pm 3.06$ \\
\hline
\end{tabular}

Values are presented as the mean \pm standard error of the mean. $* * \mathrm{P}<0.01, * \mathrm{P}<0.05$ vs. previous intervention.

R5 and R20, respiratory resistance at $5 \mathrm{~Hz}$ and $20 \mathrm{~Hz}$, respectively; X5, respiratory reactance at $5 \mathrm{~Hz}$; Fres, resonant frequency; ALX, low-frequency reactance area. 
Table 4. Pulmonary function test measurements before and after treatment

\begin{tabular}{|c|c|c|c|c|c|c|}
\hline & \multicolumn{2}{|c|}{ Whole obstruction $(n=12)$} & \multicolumn{2}{|c|}{ Tracheal obstruction $(n=6)$} & \multicolumn{2}{|c|}{ Bronchial obstruction $(n=6)$} \\
\hline & Before & After & Before & After & Before & After \\
\hline SVC, L & $2.76 \pm 0.31$ & $3.15 \pm 0.31^{*}$ & $3.06 \pm 0.52$ & $3.37 \pm 0.47$ & $2.47 \pm 0.34$ & $2.92 \pm 0.42$ \\
\hline SVC, \% predicted & $79.93 \pm 7.78$ & $91.48 \pm 7.96^{*}$ & $89.28 \pm 11.19$ & $99.32 \pm 9.80$ & $79.93 \pm 7.78$ & $91.48 \pm 7.96$ \\
\hline IC (L) & $1.89 \pm 0.23$ & $2.08 \pm 0.24$ & $2.06 \pm 0.39$ & $2.45 \pm 0.35^{* *}$ & $1.72 \pm 0.25$ & $1.71 \pm 0.27$ \\
\hline FVC, L & $2.70 \pm 0.31$ & $3.09 \pm 0.28 *$ & $2.98 \pm 0.53$ & $3.27 \pm 0.46$ & $2.41 \pm 0.32$ & $2.92 \pm 0.37$ \\
\hline FVC, \% predicted & $80.32 \pm 7.82$ & $92.85 \pm 7.37^{*}$ & $89.83 \pm 11.65$ & $99.67 \pm 9.57$ & $70.80 \pm 9.87$ & $86.03 \pm 11.36$ \\
\hline $\mathrm{FEV}_{1}, \mathbf{L}$ & $1.50 \pm 0.19$ & $2.08 \pm 0.22 *$ & $1.48 \pm 0.27$ & $2.08 \pm 0.27^{* *}$ & $1.53 \pm 0.30$ & $2.07 \pm 0.37$ \\
\hline FEV $_{1}, \%$ predicted & $55.45 \pm 7.04$ & $76.83 \pm 7.35^{* *}$ & $56.43 \pm 10.53$ & $79.03 \pm 7.66^{* *}$ & $54.47 \pm 10.32$ & $74.62 \pm 13.31$ \\
\hline FEV $_{1} /$ FVC, \% & $58.27 \pm 5.69$ & $68.27 \pm 4.39 *$ & $54.27 \pm 9.76$ & $67.02 \pm 6.90$ & $62.27 \pm 6.39$ & $69.52 \pm 6.04$ \\
\hline MMF, L/s & $1.03 \pm 0.22$ & $1.52 \pm 0.23 *$ & $1.08 \pm 0.35$ & $1.47 \pm 0.24$ & $0.98 \pm 0.29$ & $1.58 \pm 0.41$ \\
\hline MMF, \% predicted & $33.25 \pm 7.18$ & $49.09 \pm 6.74^{*}$ & $35.75 \pm 11.97$ & $48.95 \pm 7.58$ & $30.75 \pm 8.99$ & $49.23 \pm 11.92$ \\
\hline PEFR, L/s & $2.98 \pm 0.41$ & $4.21 \pm 0.54 *$ & $2.53 \pm 0.50$ & $4.23 \pm 0.62$ & $3.44 \pm 0.64$ & $4.20 \pm 0.94$ \\
\hline PEFR, \% predicted & $42.07 \pm 6.62$ & $57.42 \pm 6.93^{*}$ & $38.33 \pm 10.45$ & $58.65 \pm 6.88$ & $45.80 \pm 8.82$ & $56.18 \pm 12.79$ \\
\hline V50, L/s & $1.30 \pm 0.25$ & $1.93 \pm 0.30 * *$ & $1.30 \pm 0.40$ & $1.93 \pm 0.39 * *$ & $1.29 \pm 0.34$ & $1.93 \pm 0.49^{* *}$ \\
\hline V50, \% predicted & $36.56 \pm 7.14$ & $54.80 \pm 8.12^{* *}$ & $37.70 \pm 12.07$ & $56.27 \pm 11.26^{* *}$ & $35.42 \pm 8.84$ & $53.35 \pm 12.76^{* *}$ \\
\hline V25, L/s & $0.47 \pm 0.10$ & $0.68 \pm 0.10$ & $0.50 \pm 0.15$ & $0.64 \pm 0.09$ & $0.44 \pm 0.16$ & $0.71 \pm 0.19$ \\
\hline V25, \% predicted & $36.32 \pm 7.85$ & $51.56 \pm 6.47$ & $39.08 \pm 11.46$ & $50.17 \pm 5.01$ & $33.55 \pm 11.70$ & $52.95 \pm 12.59$ \\
\hline
\end{tabular}

Values are presented as the mean \pm standard error of the mean. ${ }^{*} \mathrm{P}<0.01, * \mathrm{P}<0.05$ vs. before intervention.

VC, vital capacity; FVC, forced vital capacity; FEV1, forced expiratory volume in $1 \mathrm{~s}$; MMF, maximum mid-expiratory flow rate; PEFR, peak expiratory flow rate. 